\title{
Development And Validation Of A Simple Model For Detection Of Early Hepatocellular Carcinoma In A Liver Cirrhosis Cohort
}

This article was published in the following Dove Press journal: Cancer Management and Research

\author{
Tao $\mathrm{Li}^{1, *}$ \\ Hongguang $\mathrm{Li}^{2, *}$ \\ Aihua Wang ${ }^{3}$ \\ Xiaoyan Su' \\ Jingfang Zhao' \\ Yi Cui ${ }^{1}$ \\ Jun $\mathrm{Liu}^{4}$ \\ Jinhua $\mathrm{Hu}^{\prime}$ \\ 'Department of Gastroenterology, \\ Shandong Provincial Hospital Affiliated to \\ Shandong First Medical University, Jinan, \\ People's Republic of China; ${ }^{2}$ Shandong \\ University, Department of Hepatobiliary \\ Surgery, Shandong Provincial Hospital \\ Affiliated to Shandong First Medical \\ University, Jinan, People's Republic of \\ China; ${ }^{3}$ Department of Gastroenterology, \\ Linyi People's Hospital, Linyi, Shandong, \\ People's Republic of China; ${ }^{4}$ Department \\ of Liver Transplantation and \\ Hepatobiliary Surgery, Shandong \\ Provincial Hospital Affiliated to Shandong \\ First Medical University, Jinan, People's \\ Republic of China \\ *These authors contributed equally to \\ this work
}

Correspondence: Jinhua $\mathrm{Hu}$ Department of Gastroenterology, Provincial Hospital Affiliated to Shandong University, 324, Jing 5 Road, Jinan 25002I, Shandong Province, People's Republic of China

Tel +86-53I-68773299

Email15665783658@I63.com

Jun Liu

Department of Liver Transplantation and Hepatobiliary Surgery, Shandong

Provincial Hospital Affiliated to Shandong First Medical University, Jing 5 Road, Jinan,

People's Republic of China

Tel +86-53l-68773299

Email dr_liujun1967@yeah.net

\begin{abstract}
Aim: We aimed to develop a simple model combining protein induced by vitamin $\mathrm{K}$ antagonist-II (PIVKA-II) and alpha-fetoprotein (AFP) to detect early hepatocellular carcinoma (HCC) in liver cirrhosis (LC) patients.
\end{abstract}

Method: One hundred and sixty-nine newly diagnosed early HCC patients and 242 LC patients without HCC were enrolled in the current case-control study. All subjects were randomly divided into analysis group and validation group. Serum levels of PIVKA-II, AFP and other laboratory parameters were detected. Chi-squared test, $t$-test and logistic regression were employed in statistical analysis.

Results: PIVKA-II level in early HCC was significantly higher than that in LC $(90.97 \mathrm{mAU} / \mathrm{mL}$ vs $18.00 \mathrm{mAU} / \mathrm{mL}, P<0.01)$, as well as AFP level $(15.00 \mathrm{ng} / \mathrm{mL}$ vs $2.00 \mathrm{ng} / \mathrm{mL}, P<0.01)$ in analysis groups. Multivariate analysis showed that PIVAK-II, AFP, gender, and age were independent risk factors for early HCC detection among LC patients. A logistic regression model and a simple model combining PIVKA and AFP were conducted to detect early HCC. The ROC curve showed that among analysis groups, the area under the ROC curve (AUROC) of the logistic regression model and the simple model were 0.96 (95\% CI 0.94-0.98) and 0.94 (95\% CI $0.92-0.97)$, respectively. At a cut-off value of 56.03 the sensitivity and specificity of the simple model were $81.1 \%$ and $91.4 \%$, respectively. In the validation group, the sensitivity and specificity of the simple model was $82.4 \%$ and $94.2 \%$, respectively. The two models are comparable statistically for early HCC detection, but the logistic regression requires complex calculation.

Conclusion: The present study indicates that the simple model combining PIVKA-II and AFP has comparable diagnostic efficiency in contrast to the logistic model but is easy to use clinically. It might be helpful for early HCC detection among liver cirrhosis patients.

Keywords: hepatocellular carcinoma, PIVKA-II, AFP, liver cirrhosis

\section{Introduction}

Hepatocellular carcinoma (HCC) is the sixth most common cancer and the second most common cause of cancer mortality world-wide. It usually develops from chronic liver diseases such as chronic hepatitis and cirrhosis. ${ }^{1,2}$ Despite continuous improvement in recent years, the overall one-year and overall five-year survival rates remain at $47 \%$ and $20 \%$, respectively, which may be due to the advanced stage at the time of diagnosis. ${ }^{1,3}$ On the contrary, the median survival of HCC diagnosed at early stage could achieve about $70 \%$ at 5 years treated with surgical resection. ${ }^{4}$

In order to detect early HCC among liver cirrhosis patients, a semi-annual surveillance program with abdominal ultrasound (US) and serum alpha-fetoprotein 
(AFP) level were recommended. ${ }^{5,6}$ For that, at an early stage HCC patients could achieve five-year survival rates of $70 \%$ with liver resection, while those with advanced or late stage such as BCLC C stage are not eligible for resection and only have a median survival of less than one year. ${ }^{4,7}$ However, it is difficult to detect early HCC by US examination due to its complicated background in cirrhotic liver, and the sensitivity of US in HCC detection was only $63 \%$ in liver cirrhosis patients according to a meta-analysis study. ${ }^{8,9}$ For another, a number of HCC patients are AFPnegative and serum AFP level has limited sensitivity for the diagnosis of early HCC, as small, well-differentiated HCC is frequently accompanied by normal serum AFP level. ${ }^{10} \mathrm{At}$ the commonly used cut-off $(20 \mathrm{ng} / \mathrm{mL})$, the sensitivity of AFP is unsatisfactory, especially for the detection of early HCC. ${ }^{11}$ Therefore, more biomarkers of early HCC are needed to complement US and AFP.

Protein induced by vitamin K antagonist-II (PIVKAII), also known as Des- $\gamma$-carboxyprothrombin (DCP), is an abnormal prothrombin protein produced by HCC cells, and has been found to possess several biological activities in the development of HCC. ${ }^{12}$ For instance, PIVKA-II could bind with cellular mesenchymal-epithelial transition factor (c-Met), which is an initial event in the progression of HCC. ${ }^{13}$ Moreover, PIVKA-II plays an important role in angiogenesis of HCC through the activation of the KDRPLC- $\gamma$-MAPK signaling pathway. ${ }^{14,15}$ According to a recent study on early HCC in France, PIVKA-II demonstrated more efficient than AFP in the diagnosis of early HCC and could be used as a predictor of microvascular invasion (MVI). ${ }^{16}$ Moreover, in another research in China, PIVKA-II exhibited the best diagnostic performance in the early diagnosis of HCC among alpha-fetoprotein (AFP), lens culinaris agglutinin-reactive AFP (AFP-L3), squamous cell carcinoma antigen, and centromere protein F autoantibody. ${ }^{17}$ Therefore, the Japan Society of Hepatology (JSH) recommends PIVKA-II as an important surveillance method for HCC in at-risk populations such as liver cirrhosis and chronic hepatitis B (CHB) patients. $^{18,19}$

Based upon the above findings, we hypothesized that PIVKA-II may play a critical role in early HCC detection among liver cirrhosis (LC) patients. In order to verify the hypothesis, we investigated the diagnostic value of PIVAKA-II and constructed two models combining PIVKA-II and AFP to detect early HCC among patients with LC.

\section{Patients And Methods}

\section{Patients}

Between October 2017 and March 2019, a total of 411 consecutive patients with early HCC and LC were enrolled in this study. HCC diagnosis was based on pathology or typical radiologic results of HCC on two dynamic image examinations or one dynamic technique with serum AFP level $\geq 200 \mathrm{ng} / \mathrm{mL}$. Early HCC was diagnosed with the BCLC staging system, which was defined as a single lesion between 2 and $5 \mathrm{~cm}$, or $\leq 3$ lesions each $\leq 3 \mathrm{~cm} \cdot{ }^{20,21}$ LC was diagnosed by histology or on clinical findings with abdominal US, CT and magnetic rsonance imaging (MRI) features of blunted, nodular liver edge accompanied by splenomegaly and esophageal varices. Clinicopathologcial and laboratory data were obtained from all subjects after written informed consents were obtained.

The study protocol is consistent with the ethical guidelines of the 1975 Declaration of Helsinki and it was approved by the ethical committee of Shandong Provincial Hospital affiliated to Shandong First Medical University. Written informed consent was obtained from each subject.

\section{Serum Sample Collection And Assays}

Peripheral blood samples were collected from each subject right before surgeries or other treatments, followed by spinning and storage at $-80^{\circ} \mathrm{C}$ until laboratory tests. PIVKA-II serum levels were detected with Lumipulse $\mathrm{G}$ PIVKA-II reagent kits (FUJIREBIO Inc., Japan) on a LUMI-PULSE g1200 automatic immune analyzer according to the manufacturer's manual.

\section{Development Of Logistic Regression Model}

A formula to detect early $\mathrm{HCC}$ in L patients was developed based on the subjects of the analysis group. The standard logistic regression formula is:

$\operatorname{Logit}(\operatorname{Stex} P)=\beta 0+\beta 1 X 1+\beta 2 X 2+\ldots \ldots+\beta n X n$. "P" is the estimated probability of early HCC in the LC cohort, while " $n$ " is the number of influence factors, " $\beta$ " represents the influence coefficient, " $X$ " is the influence factor, and " $\beta 0$ " is a constant.

\section{Statistical Analysis}

Continuous variables with normal distribution examined by Kolmogorov-Smirnov test are presented as mean \pm standard deviation and were compared using Student's $t$-test, while 
categorical variables are expressed in absolute values and compared with the chi-square test. Logistic regression analysis was performed to detect independent variables of risk factors for early HCC detection. Receiver operating characteristic (ROC) curve analysis was used to measure the diagnostic accuracy of developed models for early HCC detection in LC patients. Statistical analysis was performed using SPSS version 21.0 (SPSS, Chicago, Illinois), Prism6 (GraphPad software, La Jolla, California), and R software (version 3.6.1; http://www.Rproject.org). The package used in $\mathrm{R}$ was rms. Statistical analysis was tested on two-sided settings and $P<0.05$ was considered as statistically significant.

\section{Results}

\section{Baseline Characteristics Of All Subjects}

One hundred and sixty-nine newly diagnosed early HCC patients with LC and $242 \mathrm{LC}$ patients without HCC were enrolled in the present study. The baseline characteristics of all patients are summarized in Table 1. The average age was 54.34 years, and $72.51 \%$ of the patients were male. All HCC patients were BCLC stage 0-A4, with ChildPugh A-B.

\section{Higher PIVKA-II And AFP Levels Were Detected In Early HCC Than In LC Patients}

The characteristics of the analysis group are shown in Table 2. Elevated serum PIVKA-II and AFP levels were detected in early HCC patients compared with LC patients in the analysis group. The serum level of PIVKA-II among early HCC patients was $90.97(9.7-30,766.38) \mathrm{mAU} / \mathrm{mL}$, significantly higher than that of the LC patients 18 (3.49626.34) $\mathrm{mAU} / \mathrm{mL}(P<0.01)$ in the analysis group (Figure 1$)$. The serum level of AFP among early HCC patients was 15 $(0.9-19,549.1) \mathrm{ng} / \mathrm{mL}$, significantly higher than that of the LC patients $[2.00(0.6-121.8) \mathrm{ng} / \mathrm{mL}(P<0.01)$ in the analysis group] (Figure 1).

Univariate analysis showed that a serum PIVKA-II level higher than $29.72 \mathrm{mAU} / \mathrm{mL}(\mathrm{OR}=43.74,95 \%$ CI $15.53-$ 123.19) and a serum AFP level higher than $4.05 \mathrm{ng} / \mathrm{mL}$ (OR $=15.59,95 \%$ CI 7.16-33.95) were risk factors for diagnosis of early HCC among LC patients after logarithmic transformation. According to the ROC curve, PIVKA-II presented a similar diagnostic value compared with AFP in discriminating early HCC among LC patients with the AUROC of 0.89 (95\% CI $0.85-0.93)$ and $0.90 \quad(95 \%$ CI $0.85-0.94)$,

Table I Baseline Characteristics Of The Study Population

\begin{tabular}{|l|l|l|l|l|}
\hline Variables & All Patients & Analysis Group & Validation Group & P value \\
\hline Sex F/M & $113 / 298$ & $68 / 166$ & $45 / 132$ & 0.44 \\
Age (years) & $54.34 \pm 10.83$ & $54.37 \pm 10.55$ & $54.32 \pm 11.23$ & 0.43 \\
ALT & $32(4-1138)$ & $33.5(6-1138)$ & $31(4-547)$ & 0.58 \\
AST & $36(10-1217)$ & $35(10-1217)$ & $38(13-427)$ & 0.15 \\
GGT & $34(7-1082)$ & $32(7-1082)$ & $37(11-304)$ & 0.14 \\
TBiL & $22.3(6.3-573.5)$ & $22.42(6.3-133)$ & $22.2(8-573.5)$ & 0.87 \\
ALB & $35.64 \pm 6.28$ & $35.55 \pm 6.06$ & $35.76 \pm 6.57$ & 0.11 \\
D-Dimer & $0.7(0.05-28.26)$ & $0.8(0.1-27.4)$ & $0.6(0.05-28.26)$ & 0.26 \\
PTA & $73.1 \pm 16.67$ & $71.88 \pm 17.09$ & $74.7 \pm 16.02$ & 0.16 \\
PLT (xI0 $/$ /L) & $112.12 \pm 82.37$ & $107.99 \pm 75.02$ & $117.59 \pm 91.13$ & 0.15 \\
AFP & $3.3(0.5-19,549.10)$ & $2.95(0.60-19,549.10)$ & $3.9(0.50-7679.70)$ & 0.11 \\
PIVKA-II & $25.87(3.49-30,766.38)$ & $25.18(3.49-30,766.38)$ & $27.96(4.40-26,785.97)$ & 0.52 \\
\hline Child-Pugh grade & & & & \\
A & 290 & 173 & 117 & 0.09 \\
B & 121 & 61 & 60 & \\
\hline Tumor number & 156 & 88 & 68 & \\
Solitary & 13 & 7 & 6 & 0.86 \\
Multifocal & & 15 & 10 & \\
\hline BCLC stage & 25 & 80 & 64 & 0.68 \\
0 & 144 & & & \\
A & & 80 & & \\
\hline
\end{tabular}


Table 2 Baseline Characteristics Of Analysis Group

\begin{tabular}{|c|c|c|c|c|}
\hline Variables & Analysis Group & Early HCC & Liver Cirrhosis & $P$ value \\
\hline Sex F/M & $68 / 166$ & $18 / 77$ & $50 / 89$ & 0.005 \\
\hline Age (years) & $54.37 \pm 10.55$ & $57.01 \pm 9.87$ & $52.56 \pm 10.64$ & 0.001 \\
\hline ALT & $33.5(6-1138)$ & 35 (7-728) & $33(6-1138)$ & 0.13 \\
\hline AST & $35(10-1217)$ & $36(10-1217)$ & $33(10-598)$ & 0.13 \\
\hline GGT & $32(7-1082)$ & $36(7-8 \mid 7)$ & $30(10-1082)$ & 0.12 \\
\hline TBiL & $22.42(6.30-133)$ & $23.50(6.30-75.10)$ & $21.80(6.35-133)$ & 0.72 \\
\hline ALB & $35.55 \pm 6.06$ & $38.08 \pm 5.52$ & $33.82 \pm 5.81$ & 0.83 \\
\hline D-Dimer (mg/L) & $0.80(0.10-27.4)$ & $0.48(0.1-20)$ & $0.92(0.11-27.40)$ & 0.44 \\
\hline PTA & $71.88 \pm 17.09$ & $80.73 \pm 14.96$ & $65.84 \pm 15.8$ & 0.51 \\
\hline PLT $\left(\times 10^{9} / \mathrm{L}\right)$ & $107.99 \pm 75.02$ & $1 \mid 3.09 \pm 66.97$ & $104.50 \pm 80.10$ & 0.47 \\
\hline $\operatorname{AFP}(\mathrm{ng} / \mathrm{mL})$ & $2.95(0.60-19,549.10)$ & $15(0.90-19,549.1)$ & $2(0.60-121.8)$ & $<0.01$ \\
\hline PIVKA-II (mAU/mL) & $25.18(3.49-30,766.38)$ & $90.97(9.7-30,766.38)$ & $18(3.49-626.34)$ & $<0.01$ \\
\hline \multicolumn{5}{|l|}{ Child-Pugh class } \\
\hline A & 173 & 71 & 102 & 0.82 \\
\hline B & 61 & 24 & 37 & \\
\hline
\end{tabular}

respectively. The sensitivity of PIVKA-II was $84.20 \%$, the specificity of PIVKA-II $82.00 \%$, while the sensitivity of AFP was $85.30 \%$, and the specificity $85.60 \%$ (Figure 2 ).

Moreover, multivariate analysis also showed that male gender $(\mathrm{OR}=2.70,95 \% \mathrm{CI} 1.01-7.16)$ and age $(\mathrm{OR}=1.04$, 95\% CI 1.00-1.08) were independent risk factors for diagnosis of early HCC among LC patients (Table 3 ).

\section{Development Of A Logistic Regression Model And A Simple Model In The Analysis Group}

A total of 95 early HCC with LC and 139 LC patients in the analysis group were enrolled to build a logistic regression model combining PIVKA-II, AFP, gender, and age. The (a)

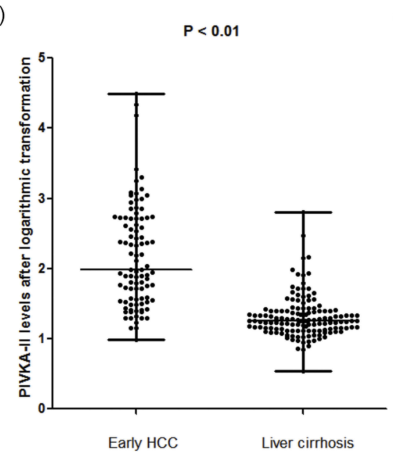

(b)

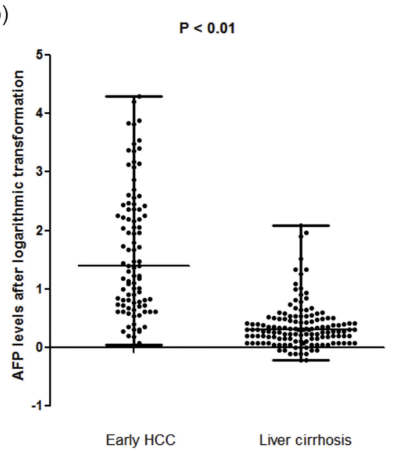

Figure I Difference of PIVKA and AFP plasma levels after logarithmic transformation between early HCC patients and liver cirrhosis in the analysis group. (A) Serum level of PIVKA-II among early HCC patients was 90.97 (9.7-30,766.38) $\mathrm{mAU} / \mathrm{mL}$, significantly higher than that of LC patients at 18 (3.49-626.34) $\mathrm{mAU} /$ $\mathrm{mL}(P<0.0 \mathrm{I})$ in analysis group. (B) Serum level of AFP among early HCC patients was I5 $(0.9-19,549.1) \mathrm{ng} / \mathrm{mL}$, significantly higher than that of LC patients at 2.00 $(0.6-121.8) \mathrm{ng} / \mathrm{mL}(P<0.01)$ in analysis group.

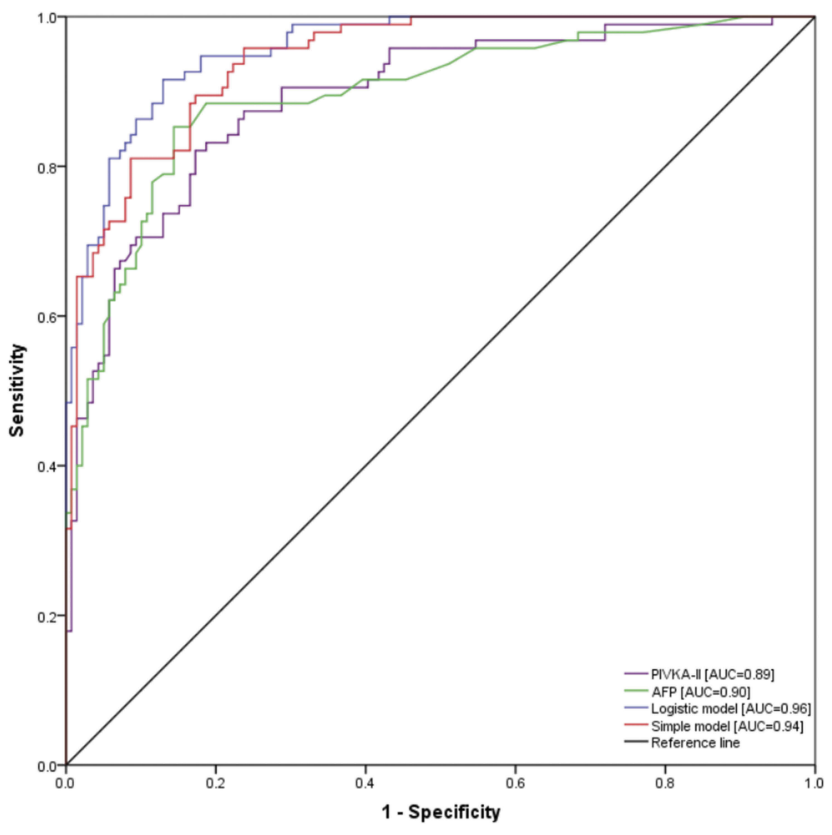

Figure 2 Diagnostic values of AFP (green), PIVKA-II (purple), the logistic regression model (blue), and the simple model (red) for early HCC detection among ILC patients in the analysis group.

results of univariate logistic analysis and multivariate logistic analysis are presented in Table 3. The final logistic regression model for predicting early $\mathrm{HCC}$ with liver cirrhosis was: Logit $(\mathrm{P})=(-10.13)+3.12(\mathrm{PIVKA})+2.54$ $(\mathrm{AFP})+0.73$ (gender) +0.04 (age). The performance of the model was good with an AUROC of 0.96 (95\% CI $0.94-$ $0.98, P<0.01$ ) (Figure 2). At a cut-off probability of 0.31 [Logit $(P)=-0.80]$, the sensitivity and specificity of the model for early HCC detection were $87.4 \%$ and $87.8 \%$, respectively. 
Table 3 Univariate And Multivariate Analyses Of All Characteristics In Analysis Group

\begin{tabular}{|c|c|c|c|c|}
\hline \multirow[t]{2}{*}{ Characteristic } & \multicolumn{2}{|c|}{ Univariate Analysis } & \multicolumn{2}{|c|}{ Multivariate Analysis } \\
\hline & OR $(95 \% \mathrm{CI})$ & $P$ value & OR (95\% CI) & $P$ value \\
\hline Sex F/M & $2.11(1.15,3.85)$ & 0.02 & $2.70(1.01,7.16)$ & 0.047 \\
\hline Age (years) & $1.04(1.01,1.06)$ & $<0.01$ & $1.04(1.00,1.08)$ & 0.031 \\
\hline ALT & $1.00(0.99,1.01)$ & 0.13 & - & \\
\hline AST & $0.99(0.98,1.02)$ & 0.17 & - & \\
\hline GGT & $1.00(0.99,1.01)$ & 0.28 & - & \\
\hline TBiL & $0.99(0.98,1.00)$ & 0.33 & - & \\
\hline ALB & $0.89(0.76,1.14)$ & 0.27 & - & \\
\hline D-Dimer (mg/L) & $1.04(0.98,1.11)$ & 0.21 & - & \\
\hline PTA & $0.96(0.91,1.05)$ & 0.14 & - & \\
\hline PLT $\left(\times 10^{9} / L\right)$ & $1.00(0.99,1.01)$ & 0.39 & - & \\
\hline $\operatorname{AFP}(\mathrm{ng} / \mathrm{mL})$ & $1.04(1.02,1.06)$ & $<0.01$ & $1.03(1.01,1.05)$ & 0.001 \\
\hline PIVKA-II (mAU/mL) & $1.02(1.01,1.03)$ & $<0.01$ & $1.02(1.01,1.03)$ & $<0.01$ \\
\hline Child-Pugh class & $0.88(0.76,1.10)$ & 0.37 & - & - \\
\hline
\end{tabular}

According to the multivariate analysis, PIVKA and AFP were two critical independent risk factors for detection of early HCC in LC patients. Therefore, we developed a simple model: PIVKA+AFP, the performance of which was good with AUROC of $0.94(95 \%$ CI $0.92-0.97, P<0.01)$ (Figure 2). At a cut-off value of 56.03 the sensitivity and specificity of the model for early HCC detection was $81.1 \%$ and $91.4 \%$, respectively.

\section{Validation Of The Two Models For Early HCC Detection In Liver Cirrhosis Patients}

The characteristics of the validation group are shown in Table 4. To validate the diagnostic value of the logistic regression model in detection of early HCC in LC patients, a ROC curve was conducted in the validation group. The AUROC of the logistic regression model was 0.97 (95\% CI $0.94-0.99, P<0.01$ ) (Figure 3 ). The sensitivity and specificity of the cut-off value Logit $(\mathrm{P})=-0.80$ were $89.30 \%$ and $93.1 \%$, respectively. The mistake diagnostic rate was $6.9 \%$, the omission diagnostic rate $10.7 \%$, the total consist rate $91.53 \%$. The Concordance (C) index value of the logistic model was 0.97 and the calibration curve demonstrated good agreement between predictions and actual observations (Figure 4).

The AUROC of the simple model: PIVKA-II + AFP in the validation group was $0.95(95 \% \mathrm{CI} 0.92-0.98, P<0.01)$

Table 4 Baseline Characteristics Of Validation Group

\begin{tabular}{|c|c|c|c|c|}
\hline Variables & Validation Group & Early HCC & Liver Cirrhosis & $P$ value \\
\hline Sex $F / M$ & $45 / 132$ & $9 / 65$ & $36 / 67$ & 0.001 \\
\hline Age (years) & $54.32 \pm 11.23$ & $56.24 \pm 10.70$ & $52.94 \pm 11.46$ & 0.04 \\
\hline ALT & $31(4-547)$ & $34.05(4-547)$ & $28(6-252)$ & 0.19 \\
\hline AST & $38(13-427)$ & $39.5(13-252)$ & $36(13-427)$ & 0.84 \\
\hline GGT & $37(11-304)$ & $42.5(14-96)$ & $34(\mathrm{II}-304)$ & 0.06 \\
\hline TBiL & $22.2(8-573.5)$ & $20.68(8.67-67.70)$ & $22.80(8.00-573.5)$ & 0.23 \\
\hline ALB & $35.76 \pm 6.57$ & $35.13 \pm 5.73$ & $36.22 \pm 7.11$ & 0.26 \\
\hline D-Dimer (mg/L) & $0.6(0.05-28.26)$ & $0.4 \mid(0.07-1 \mathrm{I} .44)$ & $0.69(0.05-28.26)$ & 0.17 \\
\hline PTA & $74.7 \pm 16.02$ & $72.64 \pm 13.29$ & $76.19 \pm 17.64$ & 0.15 \\
\hline PLT $\left(\times 10^{9} / L\right)$ & $117.59 \pm 91.13$ & $|27.0| \pm 59.67$ & $110.82 \pm 108.03$ & 0.20 \\
\hline $\operatorname{AFP}(\mathrm{ng} / \mathrm{mL})$ & $3.9(0.50-7679.70)$ & $20(1.2-7679.7)$ & $2.5(0.50-16.8)$ & $<0.01$ \\
\hline PIVKA-II (mAU/mL) & $27.96(4.40-26,785.97)$ & $108.32(4.4-26,785.97)$ & $20.14(5.00-237.00)$ & $<0.01$ \\
\hline \multicolumn{5}{|l|}{ Child-Pugh class } \\
\hline A & 110 & 47 & 63 & 0.88 \\
\hline B & 67 & 27 & 40 & \\
\hline
\end{tabular}




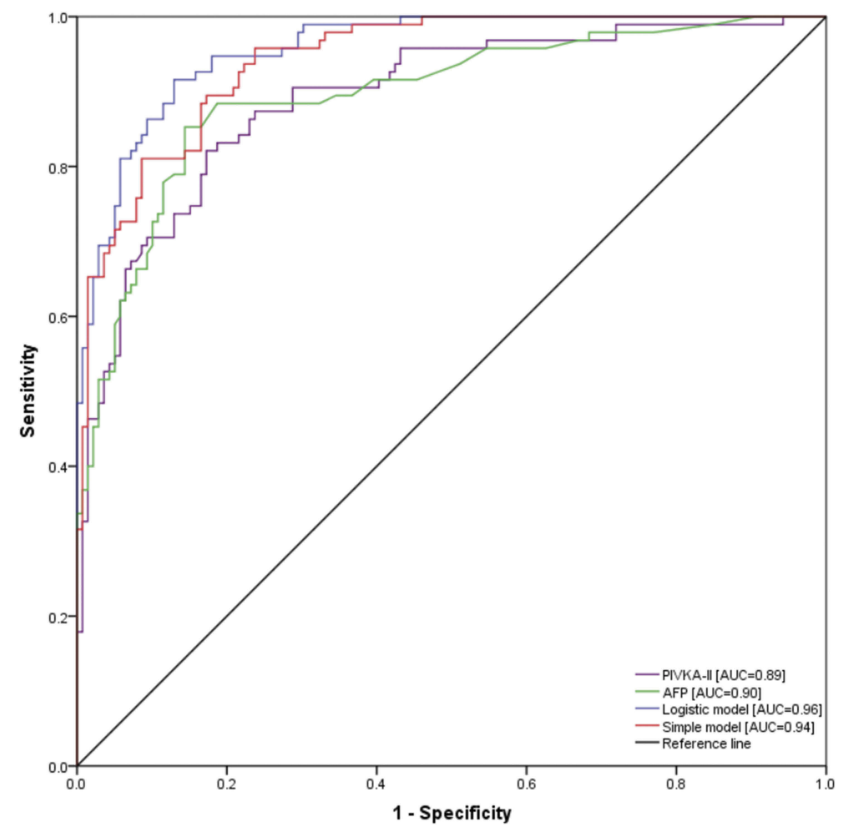

Figure 3 Diagnostic values of the logistic regression model and the simple model for early HCC detection among LC patients in the validation group.

(Figure 3). The sensitivity and specificity of the cut-off value of 56.03 was $82.4 \%$ and $94.2 \%$, respectively. The mistake diagnostic rate was $5.8 \%$, the omission diagnostic rate $17.6 \%$, the total consist rate $89.27 \%$. The $\mathrm{C}$ index value of the simple model was 0.95 and the calibration curve demonstrated good agreements between predictions and actual observations (Figure 4).

(a)

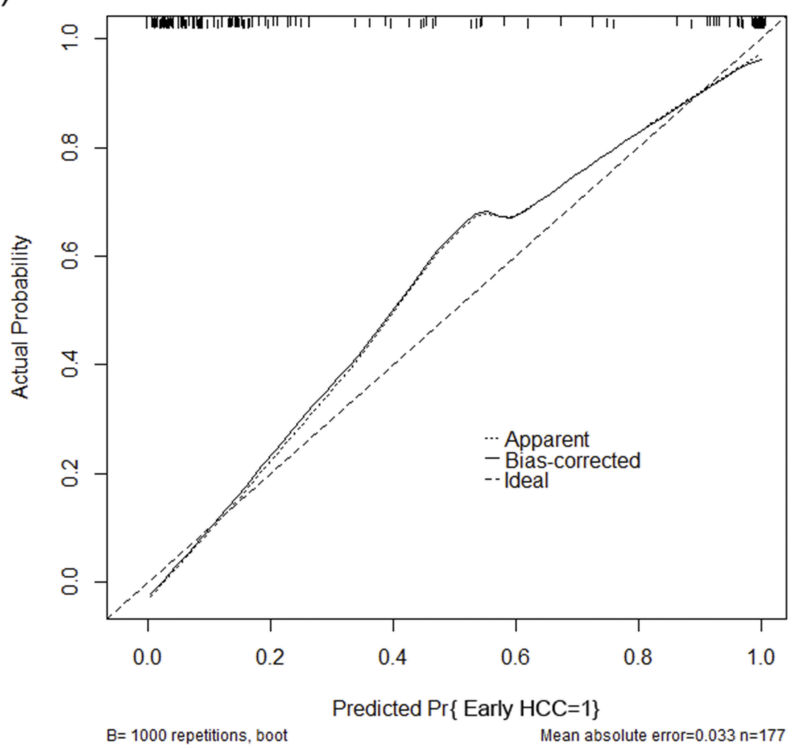

\section{Discussion}

Diagnosis of HCC at an early stage is critical for the fiveyear survival rate of HCC patients. Recent studies have indicated that diagnostic accuracy of US and AFP is limited in early $\mathrm{HCC}$ detection, especially among LC patients. ${ }^{22}$ Therefore, it is a great challenge to identify novel biomarkers or models for early-stage HCC diagnosis.

In the current study, we demonstrated that the serum PIVKA-II level was significantly higher in early HCC patients compared with LC patients, which is consistent with a recent study in a French cohort. ${ }^{16}$ These findings indicate that PIVKA-II may play a crucial role in the diagnosis of early $\mathrm{HCC}$ and hepatocarcinogenesis. Indeed, Wang et al. showed that serum PIVKA-II level could be a potential marker for early diagnosis and improve assessment of tumor prognosis. ${ }^{23}$ Moreover, PIVKA-II could promote HCC growth and metastasis through interaction with human vascular endothelial cells. ${ }^{12,24}$ Univariate analysis in our study shows that both PIVKA-II and AFP are important predictors for early $\mathrm{HCC}$ detection. We further compared the value of PIVKA and AFP in early HCC detection by ROC curve and found that PIVKA-II (AUROC $=0.89$ ) was not superior to AFP (AUROC = $0.90)$. In contrast to our findings, the above study in France showed that both PIVKA-II and AFP were efficient for the diagnosis of early HCC, but PIVKA gave a better performance than AFP for early HCC diagnosis with an

(b)

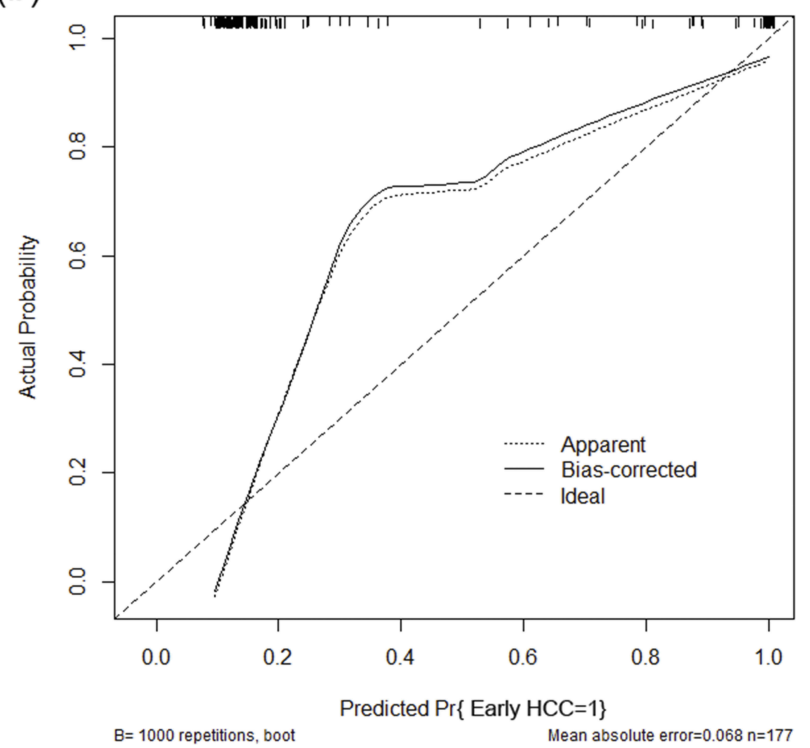

Figure 4 Validity of the predictive performance of the logistic model $(\mathbf{A})$ and the simple model $(\mathbf{B})$ in estimating the probabilities of early HCC in the validation group $(n=177)$. 
AUROC $0.81 .^{16}$ This difference may be due to our early HCC patients all having a background of LC or different geographical origin.

We hypothesized that PIVKA-II and AFP may be complementary in early $\mathrm{HCC}$ detection. Therefore, both serum levels of PIVKA-II and AFP were employed in combination to develop a logistic regression and a simple model. The logistic regression model conducted in our study possesses an AUROC of 0.96 , a sensitivity of $87.4 \%$, and a specificity of $87.8 \%$. Consistent with our results, a model that combined AFP and PIVKA-II, age, and gender developed by Random Forest (RF) classification showed a good AUROC of 0.95 $(0.95,95 \%$ CI $0.93-0.98)$, with a sensitivity of $93 \%$ and a specificity of $84 \%$ for early HCC detection in a recent study carried out in the USA. ${ }^{25}$ In another recent research in liver cirrhosis patients, Patel et al. derived a multivariate logistic regression model incorporating AFP and FIB-4 (a marker of fibrosis) for early HCC detection among LC patients, and found that the model had good discriminatory power with c-statistics of $0.78 .^{26}$ In contrast to our findings, Chen et al. also demonstrated a multimarker algorithm explored by logistic regression models consisted of PIVKA-II and AFP, which showed good ability in discriminating early $\mathrm{HCC}$ among LC AUROC of 0.81, while the AUROC of our model is $0.93 .{ }^{17}$ The different findings between our research and the above study may be due to our early HCC patients being all accompanied by liver cirrhosis.

Although the above logistic models demonstrate great value in early HCC diagnosis, they are not easy to use in clinical practice. Therefore, we developed an easy-to-use model: PIVKA + AFP, which could achieve an AUROC of 0.94 , a sensitivity of $81.1 \%$, a specificity of $91.4 \%$, at a cut-off value of 56.03 for early HCC detection among liver cirrhosis. In line with our findings, a nested case-control study also combined PIVKA-II and AFP and showed that the sensitivity for early $\mathrm{HCC}$ detection achieved about $91 \%$ at month 0 , and $73 \%$ at month 12 , and the researcher concluded that the two biomarkers are needed to complement ultrasound in early $\mathrm{HCC}$ detection. ${ }^{27}$ According to the current study, we consider that the simple model is comparable with the logistic model in statistics, but it is easy to use clinically. Given the retrospective nature of our study, it has some limitations. For instance, it was limited by possible unexpected confounders or missing data and it was a single-center study.

In conclusion, the diagnosis of early HCC is crucial and novel models are urgently needed in clinical practice. Our study suggests that a simple model of PIVKA-II + AFP could improve the accuracy of early HCC detection among liver cirrhosis patients. Further studies are necessary to validate our simple model in multicenters and more subjects.

\section{Ethics Approval And Consent To Participate}

This study was approved by the institutional ethics committee of Provincial Hospital affiliated to Shandong First Medical University. Written informed consent was obtained from each subject.

\section{Availability Of Data And Materials}

The authors can confirm all relevant data are included in the article and materials are available on request.

\section{Author Contributions}

Jinhua $\mathrm{Hu}$ and Jun Liu designed the study, Yi Cui and Jingfang Zhao edited the final version of the manuscript; Tao Li and Hongguang Li conducted the sample analysis and drafted the paper; Aihua Wang and Xiaoyan Su contributed to the statistical analyses; all authors read and approved the final manuscript. All authors contributed to data analysis, drafting or revising the article, gave final approval of the version to be published, and agree to be accountable for all aspects of the work.

\section{Disclosure}

The authors report no conflicts of interest in this work.

\section{References}

1. Grandhi MS, Kim AK, Ronnekleiv-Kelly SM, Kamel IR, Ghasebeh MA, Pawlik TM. Hepatocellular carcinoma: from diagnosis to treatment. Surg Oncol. 2016;25:74-85. doi:10.1016/j.suronc.2016.03.002

2. Shim JJ, Oh CH, Kim JW, Lee CK, Kim BH. Liver cirrhosis stages and the incidence of hepatocellular carcinoma in chronic hepatitis B patients receiving antiviral therapy. Scand $J$ Gastroenterol. 2017;52:1029-1036. doi:10.1080/00365521.2017.1335773

3. Altekruse SF, McGlynn KA, Reichman ME. Hepatocellular carcinoma incidence, mortality, and survival trends in the United States from 1975 to 2005. J Clin Oncol. 2009;27:1485-1491. doi:10.1200/ JCO.2008.20.7753

4. Llovet JM, Zucman-Rossi J, Pikarsky E, et al. Hepatocellular carcinoma. Nat Rev Dis Primers. 2016;2:16018. doi:10.1038/nrdp.2016.18

5. Singal AG, Pillai A, Tiro J. Early detection, curative treatment, and survival rates for hepatocellular carcinoma surveillance in patients with cirrhosis: a meta analysis. Ann Intern Med. 2014;161:261-269. doi:10.7326/M14-0558

6. Heimbach JK, Kulik LM, Finn RS, et al. AASLD guidelines for the treatment of hepatocellular carcinoma. Hepatology. 2018;67:358-380. doi: $10.1002 /$ hep. 29086

7. Mokdad AA, Hester CA, Singal AG, Yopp AC. Management of hepatocellular in the United States. Chin Clin Oncol. 2017;6:21. doi: $10.21037 / \mathrm{cco}$ 
8. Singal A, Volk M, Waljee A, et al. Meta-analysis:surveillance with ultrasound for early stage hepatocellular carcinoma in patients with cirrhosis. Aliment Pharmacol Ther. 2009;30:37-47. doi:10.1111/ apt.2009.30.issue-1

9. Shang S, Plymoth A, Ge S, et al. Identification of osteopontin as a novel marker for early hepatocellular carcinoma. Hepatology. 2012;55:483-490. doi:10.1002/hep.24703

10. Wang FH, Yip YC, Zhang M, et al. Diagnostic utility of glypican-3 for hepatocellular carcinoma on liver needle biopsy. J Clin Pathol. 2010;63:599-603. doi:10.1136/jcp.2010.075374

11. El-Serag HB, Marrero JA, Rudolph L, Reddy KR. Diagnosis and treatment of hepatocellular carcinoma. Gastroenterology. 2008;134:17521763. doi:10.1053/j.gastro.2008.02.090

12. Suzuki M, Shiraha H, Fujikawa T, et al. Des-gamma-carboxyprothrombin is a potential autologous growth factor for hepatocellular carcinoma. J Biol Chem. 2005;280:6409-6415. doi:10.1074/jbc. M406714200

13. Zhang YS, Chu JH, Cui SX, Qu XJ. Des- $\gamma$-carboxyprothrombin (DCP) as a potential autologous growth factor for the development of hepatocellular carcinoma. Cell Physiol Biochem. 2014;34:903915. doi:10.1159/000366308

14. Fujikawa T, Shiraha H, Ueda N, et al. Des-gamma-carboxyl prothrombin promoted vascular endothelial cell proliferation and migration. J Biol Chem. 2007;282:8741-8748. doi:10.1074/jbc.M609358 200

15. Matsubara M, Shiraha H, Kataoka J, et al. Des- $\gamma$-carboxyl prothrombin is associated with tumor angiogenesis in hepatocellular carcinoma. J Gastroenterol Hepatol. 2012;27:1602-1608. doi:10.1111/ jgh.2012.27.issue-10

16. Poté N, Cauchy F, Albuquerque M, et al. Performance of PIVKA-II for early hepatocellular carcinoma diagnosis and prediction of microvascular invasion. $J$ Hepatol. 2015;62:848-854. doi:10.1016/j. jhep.2014.11.005

17. Chen H, Zhang Y, Li S, et al. Direct comparison of five serum biomarkers in early diagnosis of hepatocellular carcinoma. Cancer Manag Res. 2018;10:1947-1958. doi:10.2147/CMAR
18. Kudo M, Izumi N, Kokudo N, et al. Management of hepatocellular carcinoma in Japan: consensus-based clinical practice guidelines proposed by the Japan Society of Hepatology (JSH) 2010 updated version. Dig Dis. 2011;29:339-364. doi:10.1159/000327577

19. Kudo M, Matsui O, Izumi N, et al. JSH consensus-based clinical practice guidelines for the management of hepatocellular carcinoma: 2014 update by the Liver Cancer Study Group of Japan. Liver Cancer. 2014;3:458-468. doi:10.1159/000343875

20. Song DS, Bae SH. Changes of guidelines diagnosing hepatocellular carcinoma during the last ten-year period. Clin Mol Hepatol. 2012;18:258-267. doi:10.3350/cmh.2012.18.3.258

21. Bruix J, Sherman M. Management of hepatocellular carcinoma: an update. Hepatology. 2011;53:1020-1022. doi:10.1002/hep.24199

22. Wang X, Kwak KJ, Yang Z, et al. Extracellular mRNA detected by molecular beacons in tethered lipoplex nanoparticles for diagnosis of human hepatocellular carcinoma. PLoS One. 2018;13:e0198552. doi:10.1371/journal.pone.0198552

23. Wang X, Zhang W, Liu Y, et al. Diagnostic value of prothrombin induced by the absence of vitamin $\mathrm{K}$ or antagonist-II (PIVKA-II) for early stage HBV related hepatocellular carcinoma. Infect Agent Cancer. 2017;12:47. doi:10.1186/s13027-017-0153-6

24. Fujikawa T, Shirah AH, Yamamoto K. Significance of des-gammacarboxyprothrombin production in hepatocellular carcinoma. Acta Med Okayama. 2009;63:299-304. doi:10.18926/AMO/31826

25. Hemken PM, Sokoll LJ, Yang X, et al. Validation of a novel model for the early detection of hepatocellular carcinoma. Clin Proteomics. 2019;16:2. doi:10.1186/s12014-018-9222-0

26. Patel J, Yopp A, Waljee AK, Singal AG. Development and internal validation of a model for early detection of hepatocellular carcinoma in patients with cirrhosis. J Clin Gastroenterol. 2016;50:175-179. doi:10.1097/MCG.0000000000000377

27. Lok AS, Sterling RK, Everhart JE, et al. Des-gamma-carboxy prothrombin and alpha-fetoprotein as biomarkers for the early detection of hepatocellular carcinoma. Gastroenterology. 2010;138:493-502. doi:10.1053/j.gastro.2009.10.031

\section{Publish your work in this journal}

Cancer Management and Research is an international, peer-reviewed open access journal focusing on cancer research and the optimal use of preventative and integrated treatment interventions to achieve improved outcomes, enhanced survival and quality of life for the cancer patient.
The manuscript management system is completely online and includes a very quick and fair peer-review system, which is all easy to use. Visit http://www.dovepress.com/testimonials.php to read real quotes from published authors. 